\title{
On solutions of fractional Riccati differential equations
}

\author{
Mehmet Giyas Sakar ${ }^{1}$, Ali Akgül2* (1) and Dumitru Baleanu ${ }^{3,4}$
}

\author{
"Correspondence: \\ aliakgul00727@gmail.com \\ ${ }^{2}$ Department of Mathematics, Art \\ and Science Faculty, Siirt University, \\ Siirt, 56100, Turkey \\ Full list of author information is \\ available at the end of the article
}

\begin{abstract}
We apply an iterative reproducing kernel Hilbert space method to get the solutions of fractional Riccati differential equations. The analysis implemented in this work forms a crucial step in the process of development of fractional calculus. The fractional derivative is described in the Caputo sense. Outcomes are demonstrated graphically and in tabulated forms to see the power of the method. Numerical experiments are illustrated to prove the ability of the method. Numerical results are compared with some existing methods.
\end{abstract}

Keywords: iterative reproducing kernel Hilbert space method; inner product; fractional Riccati differential equation; analytic approximation

\section{Introduction}

In this work, we present an iterative reproducing kernel Hilbert spaces method (IRKHSM) for investigating the fractional Riccati differential equation of the following form $[1,2]$ :

$$
{ }^{c} D_{0+}^{\alpha} u(\eta)=p(\eta) u^{2}(\eta)+q(\eta) u(\eta)+r(\eta), \quad 0 \leq \eta \leq T,
$$

with the initial condition

$$
u(0)=0,
$$

where $p(\eta), q(\eta), r(\eta)$ are real continuous functions, and $u(\eta) \in W_{2}^{2}[0, T]$.

The Riccati differential equation is named after the Italian nobleman Count Jacopo Francesco Riccati (1676-1754). The book of Reid [1] includes the main theories of Riccati equation, with implementations to random processes, optimal control, and diffusion problems [2].

Fractional Riccati differential equations arise in many fields, although discussions on the numerical methods for these equations are rare. Odibat and Momani [3] investigated a modified homotopy perturbation method for fractional Riccati differential equations. Khader [4] researched the fractional Chebyshev finite difference method for fractional Riccati differential equations. Li et al. [5] have solved this problem by quasi-linearization technique.

There has been much attention in the use of reproducing kernels for the solutions to many problems in the recent years [6,7]. Those papers show that this method has many

(c) The Author(s) 2017. This article is distributed under the terms of the Creative Commons Attribution 4.0 International License (http://creativecommons.org/licenses/by/4.0/), which permits unrestricted use, distribution, and reproduction in any medium, provided you give appropriate credit to the original author(s) and the source, provide a link to the Creative Commons license, and indicate if changes were made. 
outstanding advantages [8]. Cui has presented the Hilbert function spaces. This useful framework has been utilized for obtaining approximate solutions to many nonlinear problems [9]. Convenient references for this method are [10-13].

This paper is arranged as follows. Reproducing kernel Hilbert space theory is given in Section 2. Implementation of the IRKHSM is shown in Section 3. Exact and approximate solutions of the problems are presented in Section 4. Some numerical examples are given in Section 5. A summary of the results of this investigation is given in Section 6.

\section{Preliminaries}

The fractional derivative has good memory influences compared with the ordinary calculus. Fractional differential equations are attained in model problems in fluid flow, viscoelasticity, finance, engineering, and other areas of implementations.

Definition 2.1 The Riemann-Liouville fractional integral operator of order $\alpha$ is determined as [14]

$$
J_{0+}^{\alpha} y(x)=\frac{1}{\Gamma(\alpha)} \int_{0}^{x}(x-r)^{\alpha-1} y(r) d r
$$

where $\Gamma(\cdot)$ is the gamma function, $\alpha \geq 0$ and $x>0$.

Definition 2.2 The Caputo derivative of order $\alpha$ is given as [14]

$$
{ }^{c} D_{0+}^{\alpha} y(x)=\frac{1}{\Gamma(n-\alpha)} \int_{0}^{x} \frac{\partial^{n}}{\partial x^{n}} \frac{y(r)}{(x-r)^{n-\alpha}} d r
$$

where $n-1<\alpha \leq n$ and $x>0$.

We need the following properties:

(i) $J_{0+}^{\alpha c} D_{0+}^{\alpha} y(x)=y(x)-\sum_{k=0}^{m-1} y^{k}\left(0^{+}\right) \frac{x^{k}}{k !}$.

(ii) ${ }^{c} D_{0+}^{\alpha} J_{0+}^{\alpha} y(x)=y(x)$.

\section{Reproducing kernel functions}

We describe the notion of reproducing kernel Hilbert spaces, show some particular instances of these spaces, which will play an important role in this work, and define some well-known properties of these spaces in this section.

Definition 3.1 Let $S \neq \emptyset, B: S \times S \rightarrow C$ is a reproducing kernel function of the Hilbert space $H$ iff [10]

(i) $\forall \tau \in S, \quad B(\cdot, \tau) \in H$;

(ii) $\forall \tau \in S, \forall \phi \in H, \quad\langle\phi(\cdot), B(\cdot, \tau)\rangle=\phi(\tau)$. 
Definition 3.2 The inner product space $W_{2}^{2}[0, T]$ is presented as [15]

$$
\begin{aligned}
W_{2}^{2}[0, T]= & \left\{f(t) \mid f, f^{\prime} \text { are absolutely continuous }(\mathrm{AC})\right. \text { real-valued functions, } \\
& \left.f^{\prime \prime} \in L^{2}[0, T], f(0)=0\right\},
\end{aligned}
$$

where $L^{2}[0, T]=\left\{f \mid \int_{0}^{T} f^{2}(t) d t<\infty\right\}$

$$
\langle f(t), g(t)\rangle_{W_{2}^{2}[0, T]}=f(0) g(0)+f(T) g(T)+\int_{0}^{T} f^{\prime \prime}(t) g^{\prime \prime}(t) d t
$$

and

$$
\|f\|_{W_{2}^{2}}=\sqrt{\langle f, f\rangle_{W_{2}^{2}}}, \quad f, g \in W_{2}^{2}[0, T]
$$

are the inner product and norm in $W_{2}^{2}[0, T]$.

Theorem 3.1 $W_{2}^{2}[0, T]$ is an RKHS. There exist $R_{x}(t) \in W_{2}^{2}[0, T]$ for any $f(t) \in W_{2}^{2}[0, T]$ and each fixed $x \in[0, T], t \in[0, T]$, such that $\left\langle f(t), R_{x}(t)\right\rangle_{W_{2}^{2}}=f(x)$. The reproducing kernel $R_{x}(t)$ can be written as [15]

$$
R_{x}(t)= \begin{cases}R_{1}(x, t), & t \leq x, \\ R_{1}(t, x), & t>x,\end{cases}
$$

where

$$
R_{1}(x, t)=t\left((x-T) T t^{2}+x\left(2 T^{3}-3 x T^{2}+x^{2} T+6\right)\right) /\left(6 T^{2}\right) .
$$

Definition 3.3 $W_{2}^{1}[0, T]$ is given as $[15]$

$$
\begin{aligned}
& W_{2}^{1}[0, T]=\left\{f(x) \mid f \text { is AC real-valued function, } f^{\prime} \in L^{2}[0, T]\right\} \\
& \langle f(t), g(t)\rangle_{W_{2}^{1}[0, T]}=f(0) g(0)+\int_{0}^{T} f^{\prime}(t) g^{\prime}(t) d t
\end{aligned}
$$

and

$$
\|f\|_{W_{2}^{1}}=\sqrt{\langle f, f\rangle_{W_{2}^{1}}}, \quad f, g \in W_{2}^{1}[0, T]
$$

are the inner product and norm in $W_{2}^{1}[0, T]$.

$W_{2}^{1}[0, T]$ is am RKHS, and its reproducing kernel function is obtained as

$$
T_{x}(t)= \begin{cases}1+t, & t \leq x \\ 1+x, & t>x\end{cases}
$$




\section{Solutions to the fractional Riccati differential equations in RKHS}

The solution of (1)-(2) has been obtained in the RKHS $W_{2}^{2}[0, T]$. To get through with the problem, we investigate equation (1) as

$$
u(\eta)=f(\eta, u(\eta)), \quad 0<\eta<T
$$

where

$$
f(\eta, u(\eta))=\frac{1}{\Gamma(\alpha)} \int_{0}^{\eta}(\eta-\tau)^{\alpha-1}\left(p(\tau) u^{2}(\tau)+q(\tau) u(\tau)+r(\tau)\right) d \tau, \quad 0<\alpha \leq 1 .
$$

Let $L: W_{2}^{2}[0, T] \rightarrow W_{2}^{1}[0, T]$ be such that $L u(\eta)=u(\eta)$. Then $L$ is a bounded linear operator. We define $\varphi_{i}(\eta)=T_{\eta_{i}}(\eta)$ and $\psi_{i}(\eta)=L^{*} \varphi_{i}(\eta)$. By the Gram-Schmidt orthogonalization process we obtain

$$
\bar{\psi}_{i}(\eta)=\sum_{k=1}^{i} \beta_{i k} \psi_{k}(\eta) \quad\left(\beta_{i i}>0, i=1,2, \ldots\right)
$$

Theorem 4.1 If $\left\{\eta_{i}\right\}_{i=1}^{\infty}$ is dense on $[0, T]$, then $\left\{\psi_{i}(\eta)\right\}_{i=1}^{\infty}$ is a complete system of $W_{2}^{2}[0, T]$, and we have $\psi_{i}(\eta)=\left.L_{t} R_{\eta}(t)\right|_{t=\eta_{i}}$.

Proof We obtain

$$
\begin{aligned}
\psi_{i}(\eta) & =\left(L^{*} \varphi_{i}\right)(\eta)=\left\langle\left(L^{*} \varphi_{i}\right)(t), R_{\eta}(t)\right\rangle \\
& =\left\langle\varphi_{i}(t), L_{t} R_{\eta}(t)\right\rangle=\left.L_{t} R_{\eta}(t)\right|_{t=\eta_{i}}
\end{aligned}
$$

It appears that $\psi_{i}(\eta) \in W_{2}^{2}[0, T]$. For each fixed $u(\eta) \in W_{2}^{2}[0, T]$, let $\left\langle u(\eta), \psi_{i}(\eta)\right\rangle=0$ $(i=1,2, \ldots)$, which means that

$$
\left\langle u(\eta), L^{*} \varphi_{i}(\eta)\right\rangle=\left\langle L u(\cdot), \varphi_{i}(\cdot)\right\rangle=(L u)\left(\eta_{i}\right)=0 .
$$

Remark that $\left\{\eta_{i}\right\}_{i=1}^{\infty}$ is dense on $[0, T]$, and hereby $(L u)(\eta)=0$. We obtain $u \equiv 0$ by $L^{-1}$. So, the proof of Theorem 4.1 is complete.

Theorem 4.2 If $\left\{\eta_{i}\right\}_{i=1}^{\infty}$ is dense on $[0, T]$ and the solution of $(9)$ is unique, then the solution of (1)-(2) is obtained as

$$
u(\eta)=\sum_{i=1}^{\infty} \sum_{k=1}^{i} \beta_{i k} f\left(\eta_{k}, u\left(\eta_{k}\right)\right) \bar{\psi}_{i}(\eta)
$$

Proof $\left\{\bar{\psi}_{i}(\eta)\right\}_{i=1}^{\infty}$ is a complete orthonormal basis of $W_{2}^{2}[0, T]$ by Theorem 4.1. Therefore, we acquire

$$
\begin{aligned}
u(\eta) & =\sum_{i=1}^{\infty}\left\langle u(\eta), \bar{\psi}_{i}(\eta)\right\rangle_{W_{2}^{2}[0, T]} \bar{\psi}_{i}(\eta) \\
& =\sum_{i=1}^{\infty} \sum_{k=1}^{i} \beta_{i k}\left\langle u(\eta), L^{*} T_{\eta_{k}}(\eta)\right\rangle_{W_{2}^{2}[0, T]} \bar{\psi}_{i}(\eta)
\end{aligned}
$$




$$
\begin{aligned}
& =\sum_{i=1}^{\infty} \sum_{k=1}^{i} \beta_{i k}\left\langle L u(x), T_{\eta_{k}}(\eta)\right\rangle_{W_{2}^{1}[0, T]} \bar{\psi}_{i}(\eta) \\
& =\sum_{i=1}^{\infty} \sum_{k=1}^{i} \beta_{i k}\left\langle f(\eta, u(\eta)), T_{\eta_{k}}(\eta)\right\rangle_{W_{2}^{1}[0, T]} \bar{\psi}_{i}(\eta) \\
& =\sum_{i=1}^{\infty} \sum_{k=1}^{i} \beta_{i k} f\left(\eta_{k}, u\left(\eta_{k}\right)\right) \bar{\psi}_{i}(\eta) .
\end{aligned}
$$

This completes the proof.

The approximate solution $u_{n}(\eta)$ can be gained by the $n$-term intercept of the exact solution $u(\eta)$ as

$$
u_{n}(\eta)=\sum_{i=1}^{n} \sum_{k=1}^{i} \beta_{i k} f\left(\eta_{k}, u\left(\eta_{k}\right)\right) \bar{\psi}_{i}(\eta) .
$$

Remark 4.1 We notice the following two cases in order to solve equations (1)-(2) by using RKHS.

Case 1: If equation (1) is linear, that is, $p(\eta)=0$, then an approximate solution can be obtained directly from equation (12) .

Case 2: If equation (1) is nonlinear, that is, $p(\eta) \neq 0$, then an approximate solution can be obtained by using the following iterative method. According to equation (13), the exact solution of equation (1) can be denoted by

$$
u(\eta)=\sum_{i=1}^{\infty} A_{i} \bar{\psi}_{i}(\eta)
$$

where $A_{i}=\sum_{k=1}^{i} \beta_{i k} f\left(\eta_{k}, u\left(\eta_{k}\right)\right)$. In fact, $A_{i}, i=1,2, \ldots$, in (14) are unknown, and we will approximate $A_{i}$ using known $B_{i}$. For a numerical computation, we define the initial function $u_{0}\left(\eta_{1}\right)=0$ (we can choose any fixed $u_{0}\left(\eta_{1}\right) \in W_{2}^{2}[0, T]$ ) and the $n$-term approximation to $u(\eta)$ by

$$
u_{n}(\eta)=\sum_{i=1}^{n} B_{i} \bar{\psi}_{i}(\eta)
$$

where the coefficients $B_{i}$ of $\bar{\psi}_{i}(\eta)$ are given as

$$
\begin{aligned}
& B_{1}=\beta_{11} f\left(\eta_{1}, u_{0}\left(\eta_{1}\right)\right), \\
& u_{1}(\eta)=B_{1} \bar{\psi}_{1}(\eta), \\
& B_{2}=\sum_{k=1}^{2} \beta_{2 k} f\left(\eta_{k}, u_{k-1}\left(\eta_{k}\right)\right), \\
& u_{2}(\eta)=\sum_{i=1}^{2} B_{i} \bar{\psi}_{i}(\eta)
\end{aligned}
$$




$$
\begin{aligned}
& u_{n-1}(\eta)=\sum_{i=1}^{n-1} B_{i} \bar{\psi}_{i}(\eta), \\
& B_{n}=\sum_{k=1}^{n} \beta_{n k} f\left(\eta_{k}, u_{k-1}\left(\eta_{k}\right)\right) .
\end{aligned}
$$

In equation (15), we can see that the approximation $u_{n}(\eta)$ satisfies the initial condition (2). The approximate solution is computed from 15:

$$
u_{n}^{N}(\eta)=\sum_{i=1}^{N} \sum_{k=1}^{i} \beta_{i k} f\left(\eta_{k}, u_{n-1}\left(\eta_{k}\right)\right) \bar{\psi}_{i}(\eta) .
$$

Theorem 4.3 If $u(\eta) \in W_{2}^{2}[0, T]$, then there exist $E>0$ and $\|u(\eta)\|_{C[0, T]}=\max _{\eta \in[0, T]}|u(\eta)|$ such that $\left\|u^{(i)}(\eta)\right\|_{C[0, T]} \leq E\|u(\eta)\|_{W_{2}^{2}[0, T]}, i=0,1$.

Proof We obtain $u^{(i)}(\eta)=\left\langle u(t), \partial_{\eta}^{i} R_{\eta}(t)\right\rangle_{W_{2}^{2}[0, T]}$ for any $\eta, t \in[0, T]$ and $i=0,1$. Then, we get $\left\|\partial_{\eta}^{i} R_{\eta}(t)\right\|_{W_{2}^{2}[0, T]} \leq E_{i}, i=0,1$, by $R_{\eta}(t)$.

Therefore, we acquire

$$
\begin{aligned}
\left|u^{(i)}(\eta)\right| & =\mid\left\langle u(\eta),\left.\partial_{\eta}^{i} R_{\eta}(x)\right|_{W_{2}^{2}[0, T]}\right| \\
& \leq\|u(\eta)\|_{W_{2}^{2}[0, T]}\left\|\partial_{\eta}^{i} R_{\eta}(\eta)\right\|_{W_{2}^{2}[0, T]} \\
& \leq E_{i}\|u(\eta)\|_{W_{2}^{2}[0, T]}
\end{aligned}
$$

for $i=0,1$.

Thus, we get $\left\|u^{(i)}(\eta)\right\|_{C[0, T]} \leq \max \left\{E_{0}, E_{1}\right\}\|u(\eta)\|_{W_{2}^{2}[0, T]}$ for $i=0,1$. This completes the proof.

Theorem 4.4 The approximate solution $u_{n}(\eta)$ and its first derivative $u_{n}^{\prime}(\eta)$ are uniformly convergent in $[0, T]$.

Proof From Theorem 4.3, for any $\eta \in[0, T]$, we get

$$
\begin{aligned}
\left|u_{n}^{(i)}(\eta)-u^{(i)}(\eta)\right| & =\mid\left\langle u_{n}(\eta)-u(\eta),\left.\partial_{\eta}^{i} R_{\eta}(\eta)\right|_{W_{2}^{2}[0, T]}\right| \\
& \leq\left\|\partial_{\eta}^{i} R_{\eta}(\eta)\right\|_{W_{2}^{2}[0, T]}\left\|u_{n}(\eta)-u(\eta)\right\|_{W_{2}^{2}[0, T]} \\
& \leq E_{i}\left\|u_{n}(\eta)-u(\eta)\right\|_{W_{2}^{2}[0, T]}, \quad i=0,1,
\end{aligned}
$$

where $E_{0}$ and $E_{1}$ are positive constants. Hence, if $u_{n}(\eta) \rightarrow u(\eta)$ in the sense of the norm of $W_{2}^{2}[0, T]$ as $n \rightarrow \infty$, then the approximate solutions $u_{n}(\eta)$ and $u_{n}^{\prime}(\eta)$ uniformly converge to the exact solution $u(\eta)$ and its derivative $u^{\prime}(\eta)$, respectively.

\section{Numerical examples}

To give a clear overview of this technique, we give the following informative examples. All of the computations have been applied by utilizing the Maple software package. The results attained by the method are compared with the exact solution of each example and are found to be in good agreement. 
Table 1 Comparison of IRKHSM solution with other methods for Example $5.1(\alpha=1)$

\begin{tabular}{llllll}
\hline $\boldsymbol{\eta}_{\boldsymbol{i}}$ & Exact Sol. & IRKHSM & Method in [5] & Method in [16] & MHPM [3] \\
\hline 0.2 & 0.197375 & 0.197375 & 0.19738 & 0.197375 & 0.197375 \\
0.4 & 0.379949 & 0.379949 & 0.379956 & 0.379948 & 0.379944 \\
0.6 & 0.537049 & 0.537049 & 0.537061 & 0.537049 & 0.536857 \\
0.8 & 0.664037 & 0.664037 & 0.664053 & 0.664036 & 0.661706 \\
1.0 & 0.761594 & 0.761614 & 0.761618 & 0.761594 & 0.746032 \\
\hline
\end{tabular}

Table 2 Comparison of IRKHSM solution with other methods for Example $5.1(\alpha=0.9)$

\begin{tabular}{llllll}
\hline $\boldsymbol{\eta}_{\boldsymbol{i}}$ & IRKHSM & Method in [5] & Method in [16] & Method in [17] & MHPM [3] \\
\hline 0.2 & 0.238794 & 0.237652 & 0.2387891 & 0.2393 & 0.2391 \\
0.4 & 0.422593 & 0.421766 & 0.4225830 & 0.4234 & 0.4229 \\
0.6 & 0.566181 & 0.565673 & 0.5661715 & 0.5679 & 0.5653 \\
0.8 & 0.674636 & 0.674464 & 0.6746270 & 0.6774 & 0.6740 \\
1.0 & 0.754607 & 0.754632 & 0.7545890 & 0.7584 & 0.7569 \\
\hline
\end{tabular}

Table 3 Comparison of IRKHSM solution with other methods for Example $5.1(\alpha=0.75)$

\begin{tabular}{llllll}
\hline $\boldsymbol{\eta}_{\boldsymbol{i}}$ & IRKHSM & Method in [5] & Method in [16] & Method in [17] & MHPM [3] \\
\hline 0.2 & 0.310008 & 0.307359 & 0.3099755 & 0.3117 & 0.3138 \\
0.4 & 0.481693 & 0.480346 & 0.4816318 & 0.4855 & 0.4929 \\
0.6 & 0.597829 & 0.597542 & 0.5977827 & 0.6045 & 0.5974 \\
0.8 & 0.678851 & 0.679657 & 0.6788495 & 0.6880 & 0.6604 \\
1.0 & 0.736512 & 0.738213 & 0.7368368 & 0.7478 & 0.7183 \\
\hline
\end{tabular}

Example 5.1 We debate the fractional Riccati differential equation

$$
\begin{aligned}
& { }^{c} D_{0+}^{\alpha} u(\eta)=1-u^{2}(\eta), \quad 0 \leq \eta \leq T, \\
& u(0)=0 .
\end{aligned}
$$

The exact solution of (18)-(19) is given by

$$
u(\eta)=\frac{e^{2 \eta}-1}{e^{2 \eta}+1}
$$

when $\alpha=1$. Using IRKHSM for equations (18)-(19) and taking $T=1, \eta_{i}=\frac{i}{N}, i=1,2, \ldots, N$, the numerical solution $u_{n}^{N}(\eta)$ is computed. Comparison of our result with other methods at some selected grid points for $N=10, n=8$, and $\alpha=1, \alpha=0.9, \alpha=0.75$ are given in Tables $1-3$, respectively.

Example 5.2 We investigate the fractional Riccati differential equation as

$$
\begin{aligned}
& { }^{c} D_{0+}^{\alpha} u(\eta)=1+2 u(\eta)-u^{2}(\eta), \quad 0 \leq \eta \leq T, \\
& u(0)=0 .
\end{aligned}
$$

The exact solution of (21)-(22) is given as

$$
u(\eta)=1+\sqrt{2} \tanh \left(\sqrt{2} \eta+\frac{\log ((-1+\sqrt{2}) /(1+\sqrt{2}))}{2}\right)
$$


Table 4 Comparison of absolute errors for some methods for Example $5.2(\alpha=1)$

\begin{tabular}{lllll}
\hline $\boldsymbol{\eta}_{\boldsymbol{i}}$ & VIM [18] & OHAM [19] & MHPM [3] & IRKHSM \\
\hline 0.2 & $1.03 \mathrm{E}-6$ & $2.90 \mathrm{E}-4$ & $1.20 \mathrm{E}-5$ & $9.23 \mathrm{E}-5$ \\
0.4 & $3.33 \mathrm{E}-5$ & $2.50 \mathrm{E}-3$ & $3.03 \mathrm{E}-4$ & $7.35 \mathrm{E}-5$ \\
0.5 & $7.26 \mathrm{E}-5$ & $4.40 \mathrm{E}-3$ & $1.55 \mathrm{E}-3$ & $7.62 \mathrm{E}-5$ \\
0.6 & $9.98 \mathrm{E}-5$ & $5.50 \mathrm{E}-3$ & $4.69 \mathrm{E}-3$ & $7.56 \mathrm{E}-5$ \\
0.8 & $1.54 \mathrm{E}-5$ & $3.80 \mathrm{E}-3$ & $1.88 \mathrm{E}-2$ & $3.94 \mathrm{E}-5$ \\
1.0 & $3.47 \mathrm{E}-3$ & $3.40 \mathrm{E}-3$ & $3.43 \mathrm{E}-2$ & $7.12 \mathrm{E}-5$ \\
\hline
\end{tabular}

Table 5 Comparison of IRKHSM solution with other methods for Example $5.2(\alpha=0.9)$

\begin{tabular}{llllll}
\hline $\boldsymbol{\eta}_{\boldsymbol{i}}$ & IRKHSM & Method in [5] & Method in [16] & Method in [17] & MHPM [3] \\
\hline 0.2 & 0.314571 & 0.312985 & 0.314869 & - & - \\
0.4 & 0.697246 & 0.695357 & 0.697544 & - & - \\
0.5 & 0.903363 & 0.901484 & 0.903695 & 0.8621 & 0.9010 \\
0.6 & 1.107569 & 1.10576 & 1.107866 & - & - \\
0.8 & 1.477434 & 1.47606 & 1.477707 & - & - \\
1.0 & 1.765103 & 1.76417 & 1.764520 & 1.7356 & 1.8720 \\
\hline
\end{tabular}

Table 6 Comparison of IRKHSM solution with other methods for Example $5.2(\alpha=0.75)$

\begin{tabular}{lllll}
\hline $\boldsymbol{\eta}_{\boldsymbol{i}}$ & IRKHSM & Method in [5] & Method in [3] & Method in [20] \\
\hline 0.2 & 0.473076 & 0.469516 & 0.428892 & 0.584307 \\
0.4 & 0.936880 & 0.933596 & 0.891404 & 1.024974 \\
0.5 & 1.147576 & 1.14488 & 1.132763 & 1.198621 \\
0.6 & 1.333068 & 1.33098 & 1.370240 & 1.349150 \\
0.8 & 1.622033 & 1.62153 & 1.794879 & 1.599235 \\
1.0 & 1.817550 & 1.81865 & 2.087384 & 1.801763 \\
\hline
\end{tabular}

when $\alpha=1$. Using IRKHSM for equations (21)-(22) and taking $T=1, \eta_{i}=\frac{i}{N}, i=1,2, \ldots, N$, the numerical solution $u_{n}^{N}(\eta)$ is computed. The absolute errors of some methods are given in Table 4. Comparison of our result with other methods at some selected grid points for $N=13, n=6$, and $\alpha=0.9, \alpha=0.75$ are given in Tables 5 and 6 , respectively.

Example 5.3 We solve the fractional Riccati differential equation

$$
\begin{aligned}
& { }^{c} D_{0+}^{\alpha} u(\eta)=\eta^{3} u^{2}(\eta)-2 \eta^{4} u(\eta)+\eta^{5}, \\
& u(0)=0 .
\end{aligned}
$$

The exact solution of (24)-(25) is given as

$$
u(\eta)=\eta
$$

when $\alpha=1$. Using IRKHSM for equations (24)-(25) and taking $T=1, \eta_{i}=\frac{i}{N}, i=1,2, \ldots, N$, the numerical solution $u_{n}^{N}(\eta)$ is computed. Comparison of the IRKHSM solutions $u_{3}^{4}(\eta)$ for Example 5.3 with different values of $\alpha$ is given in Figure 1. The absolute error of IRKHSM solution $u_{3}^{4}(\eta)$ for Example 5.3 with $\alpha=1$ is shown in Figure 2.

\section{Conclusion}

IRKHSM were successfully implemented to get approximate solutions of the fractional Riccati differential equations. Numerical results were compared with the existing meth- 


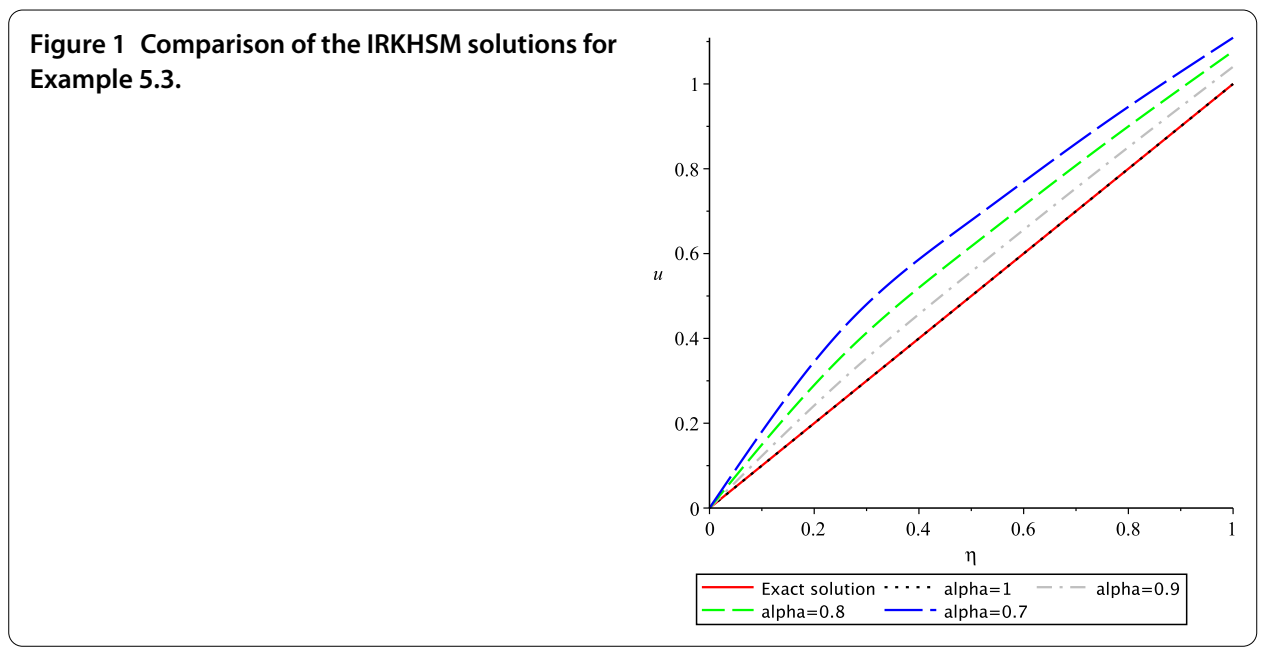

Figure 2 Absolute error of IRKHSM solution for Example 5.3.

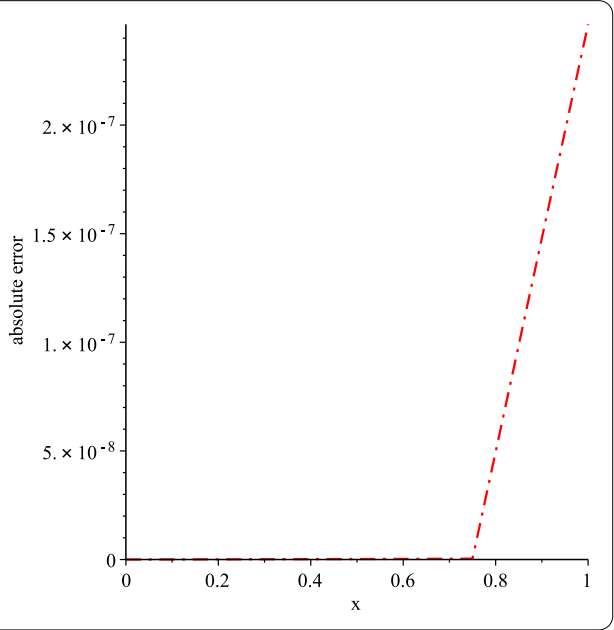

ods to prove the efficiency of the method. The IRKHSM is very powerful and accurate in obtaining approximate solutions for wide classes of the problem. The approximate solution attained by the IRKHSM is uniformly convergent. The series solution methodology can be implemented to much more complicated nonlinear equations. This method can be extended to solve the other fractional differential equations.

\section{Competing interests}

The authors declare that they have no competing interests.

Authors' contributions

All authors read and approved the final manuscript.

\section{Author details}

${ }^{1}$ Department of Mathematics, Faculty of Sciences, Yuzuncu Yil University, Van, 65080, Turkey. ${ }^{2}$ Department of Mathematics, Art and Science Faculty, Siirt University, Siirt, 56100, Turkey. ${ }^{3}$ Department of Mathematics and Computer Sciences, Art and Science Faculty, Çankaya University, Ankara, 06300, Turkey. ${ }^{4}$ Department of Mathematics, Institute of Space Sciences, Bucharest, Romania.

\section{Acknowledgements}

The authors would like to thank Mehmet EFE from Siirt University for his kind contribution.

Received: 11 August 2016 Accepted: 18 January 2017 Published online: 03 February 2017 


\section{References}

1. Reid, WT: Riccati Differential Equations. Math. Sci. Eng., vol. 86. Academic Press, New York (1972)

2. Khader, MM: Numerical treatment for solving fractional Riccati differential equation. J. Egypt. Math. Soc. 21, 32-37 (2013)

3. Odibat, Z, Momani, S: Modified homotopy perturbation method: application to quadratic Riccati differential equation of fractional order. Chaos Solitons Fractals 36(1), 167-174 (2008)

4. Khader, MM: Numerical treatment for solving fractional Riccati differential equation. J. Egypt. Math. Soc. 21(1), 32-37 (2013)

5. Li, XY, Wu, BY, Wang, RT: Reproducing kernel method for fractional Riccati differential equations. Abstr. Appl. Anal. 2014, Article ID 970967 (2014)

6. Takeuchi, T, Yamamoto, M: Tikhonov regularization by a reproducing kernel Hilbert space for the Cauchy problem for an elliptic equation. SIAM J. Sci. Comput. 31, 112-142 (2008)

7. Hon, YC, Takeuchi, T: Discretized Tikhonov regularization by reproducing kernel Hilbert space for backward heat conduction problem. Adv. Comput. Math. 34, 167-183 (2011)

8. Wang, W, Yamamoto, M, Han, B: Numerical method in reproducing kernel space for an inverse source problem for the fractional diffusion equation. Inverse Probl. 29, 095009 (2013)

9. Ghasemi, M, Fardi, M, Ghaziani, RK: Numerical solution of nonlinear delay differential equations of fractional order in reproducing kernel Hilbert space. Appl. Math. Comput. 268, 815-831 (2015)

10. Inc, M, Akgül, A: The reproducing kernel Hilbert space method for solving Troesch's problem. J. Assoc. Arab Univ. Basic Appl. Sci. 14, 19-27 (2013)

11. Inc, M, Akgül, A: Approximate solutions for MHD squeezing fluid flow by a novel method. Bound. Value Probl. 2014, 18 (2014)

12. Inc, M, Akgül, A, Geng, F: Reproducing kernel Hilbert space method for solving Bratu's problem. Bull. Malays. Math. Soc. 38, 271-287 (2015)

13. Inc, M, Akgül, A, Kilicman, A: Numerical solutions of the second order one-dimensional telegraph equation based on reproducing kernel Hilbert space method. Abstr. Appl. Anal. 2013, 13 (2013)

14. Podlubny, I: Fractional Differential Equations. An Introduction to Fractional Derivatives, Fractional Differential Equations, to Methods of Their Solution and Some of Their Applications. Mathematics in Science and Engineering, vol. 198, Academic Press, San Diego (1999). ISBN:0-12-558840-2

15. Sakar, MG: Iterative reproducing kernel Hilbert spaces method for Riccati differential equations. J. Comput. Appl. Math. 309, 163-174 (2017)

16. Yüzbaşı, Ş: Numerical solutions of fractional Riccati type differential equations by means of the Bernstein polynomials. Appl. Math. Comput. 219, 6328-6343 (2013)

17. Hosseinnia, SH, Ranjbar, A, Momani, S: Using an enhanced homotopy perturbation method in fractional differential equations via deforming the linear part. Comput. Math. Appl. 56, 3138-3149 (2008)

18. Batiha, B, Noorani, MSM, Hashim, I: Application of variational iteration method to a general Riccati equation. Int. Math. Forum 2, 2759-2770 (2007)

19. Mabood, F, Ismail, Al, Hashim, I: Application of optimal homotopy asymptotic method for the approximate solution of Riccati equation. Sains Malays. 42, 863-867 (2013)

20. Li, YL: Solving a nonlinear fractional differential equation using Chebyshev wavelets. Commun. Nonlinear Sci. Numer. Simul. 15, 2284-2292 (2010)

\section{Submit your manuscript to a SpringerOpen ${ }^{\circ}$ journal and benefit from:}

- Convenient online submission

- Rigorous peer review

- Immediate publication on acceptance

Open access: articles freely available online

- High visibility within the field

- Retaining the copyright to your article 